\title{
The Vlasov Dynamics and Its Fluctuations in the $1 / N$ Limit of Interacting Classical Particles
}

\author{
W. Braun and K. Hepp
}

Physics Department, ETH, CH-8093 Zürich, Switzerland

\begin{abstract}
For classical $N$-particle systems with pair interaction $N^{-1} \sum_{1 \leqq i \leqq j \leqq N} \phi\left(q_{i}-q_{j}\right)$ the Vlasov dynamics is shown to be the $w^{*}$-limit as $N \rightarrow \infty$. Propagation of molecular chaos holds in this limit, and the fluctuations of intensive observables converge to a Gaussian stochastic process.
\end{abstract}

\section{§ 1. Introduction}

Consider the Newtonian equation

$$
\underline{\ddot{x}}(t, a, \mu)=\int \mu(d b) \underline{F}(\underline{x}(t, a, \mu)-\underline{x}(t, b, \mu))
$$

for a particle with initial condition

$$
z(0, a, \mu)=(\underline{x}(0, a, \mu), \underline{\dot{x}}(0, a, \mu))=a=(\underline{q}, \underline{p})
$$

interacting via a regular 2-body force $\underline{F}(q)=-\underline{\nabla} \phi(q)=-\underline{F}(-q)$ with other particles having initial conditions distributed over a real Borel measure $\mu$ on $\mathbb{R}^{6}$. This framework contains the canonical dynamics of $N$ mass points

$$
\ddot{x}_{n}\left(t, \alpha_{N}\right)=\sum_{m=1}^{N} \underline{F}\left(\underline{x}_{n}\left(t, \alpha_{N}\right)-\underline{x}_{m}\left(t, \alpha_{N}\right)\right),
$$

where $1 \leqq n \leqq N$ and with initial condition $\alpha_{N}=\left(a_{1}, \ldots, a_{N}\right)$. For, let $\mu^{\alpha_{N}}(d a)$ $=\sum_{n} \delta_{a_{n}}(d a)$ and $\underline{x}\left(t, a, \mu^{\alpha_{N}}\right)$ be the solution of (1.1). Then

$$
\underline{x}_{n}\left(t, \alpha_{N}\right)=\underline{x}\left(t, a_{n}, \mu^{\alpha_{N}}\right)
$$

is the solution of (1.3). On the other hand for $\mu^{f}(d a)=f(a) d a$ the Newtonian Equation (1.1) also solves the Vlasov Equation [1]:

$$
\frac{\partial f}{\partial t}(t, a)=-\underline{p} \frac{\partial f}{\partial q}(t, a)-\frac{\partial f}{\partial \underline{p}}(t, a) \int d a^{\prime} f\left(t, a^{\prime}\right) \underline{F}\left(q-q^{\prime}\right)
$$


with initial condition $f(0, a)=f(a) \in C^{1}$, if $\int d a|f(a)| \leqq 1$. For this purpose we observe that the mapping $a \rightarrow z(t, a, \mu)$ is canonical. Then an easy calculation (2.11) shows that $f\left(t, z\left(t, a, \mu^{f}\right)\right) \equiv f(a)$ satisfies $(1.5)$.

We remark that the $N$-particle dynamics (1.3) and the Vlasov dynamics (1.5) represent disjoint specializations of (1.1) for measures $\mu^{\alpha_{N}}$ and $\mu^{f}(d a)=f(a) d a$. We expect that the Vlasov dynamics describes asymptotically the time evolution of the particle number density in $\mathbb{R}^{6}$ of the $N$-body system with initial condition $\alpha_{N}$, whenever

$$
\mu_{N}^{\alpha}=N^{-1} \sum_{n=1}^{N} \delta_{a_{n}} \stackrel{w^{*}}{\longrightarrow} \mu^{f}
$$

for $N \rightarrow \infty$. The Newtonian equations for $\mu_{N}^{\alpha}$ are equivalent to (1.3) if the 2-body force $\underline{F}$ is replaced by $N^{-1} \underline{F}$. We shall see in Section 2 that (1.6) implies $z\left(t, a, \mu_{N}^{\alpha}\right) \rightarrow z\left(t, a, \mu^{f}\right)$. Hence the Vlasov dynamics does indeed describe the continuum limit for point particle configurations where the interaction energy is scaled down to retain a finite energy per particle.

The Vlasov dynamics should also describe asymptotically the time evolution of $N$-particle systems, where the probability measure $\mu_{N}^{t}\left(d \alpha_{N}\right)=f_{N}\left(t, \alpha_{N}\right) d \alpha_{N}$ satisfies "molecular chaos" for $t=0$, i.e. $f_{N}\left(0, \alpha_{N}\right)=\prod f\left(a_{n}\right)$. This follows heuristically from the BBGKY hierarchy for the s-particle correlation functions with $N^{-1} \sum \phi_{i j}$ interaction

$$
\begin{aligned}
& f_{N}^{s}\left(t, a_{1}, \ldots, a_{s}\right)=\int d a_{s+1} \ldots d a_{N} f_{N}\left(t, \alpha_{N}\right) \\
& \frac{\partial f_{N}^{s}}{\partial t}\left(t, a_{1}, \ldots, a_{s}\right) \\
& =-\sum_{\sigma=1}^{s} \underline{p}_{\sigma} \frac{\partial f_{N}^{s}}{\partial \underline{g}_{\sigma}}\left(t, a_{1}, \ldots, a_{s}\right) \\
& \quad-\frac{1}{N} \sum_{\sigma, \tau=1}^{s} \underline{F}\left(\underline{q}_{\sigma}-\underline{q}_{\tau}\right) \frac{\partial f_{N}^{s}}{\partial \underline{p}_{\sigma}}\left(t, a_{1}, \ldots, a_{s}\right) \\
& \quad-\frac{N-s}{N} \sum_{\sigma=1}^{s} \int d a_{s+1} \underline{F}\left(q_{\sigma}-q_{s+1}\right) \frac{\partial f_{N}^{s+1}}{\partial \underline{p}_{\sigma}}\left(t, a_{1}, \ldots, a_{s+1}\right) .
\end{aligned}
$$

Formally, one obtains the "Vlasov hierarchy" for $N \rightarrow \infty$ :

$$
\begin{aligned}
& \frac{\partial f_{\infty}^{s}}{\partial t}\left(t, a_{1}, \ldots, a_{s}\right) \\
& =-\sum_{\sigma=1}^{s} \underline{p}_{\sigma} \frac{\partial f_{\infty}^{s}}{\partial \underline{q}_{\sigma}}\left(t, a_{1}, \ldots, a_{s}\right) \\
& \quad-\sum_{\sigma=1}^{s} \int d a_{s+1} \underline{F}\left(q_{\sigma}-q_{s+1}\right) \frac{\partial f_{\infty}^{s+1}}{\partial \underline{p}_{\sigma}}\left(t, a_{1}, \ldots, a_{s+1}\right) .
\end{aligned}
$$

While the BBGKY hierarchy is incompatible with molecular chaos for all times if $\nabla \phi \neq 0$, the dynamics (1.9) has factorizable solutions $f_{\infty}^{s}\left(t, a_{1}, \ldots, a_{s}\right)=\prod_{\sigma} f\left(t, a_{\sigma}\right)$, if $f(t, a)$ is a solution of the Vlasov equation. The asymptotic propagation of 
molecular chaos has been established by Kac [2], Grünbaum [3], and McKean [4] for the " $N$ molecule gas" and by Grad [5], Lanford [6] and King [7] for the Boltzmann limit. In Section 3 we shall prove the convergence of $f_{N}^{s}\left(t, a_{1}, \ldots, a_{s}\right)$ to $\prod_{\sigma=1}^{s} f\left(t, a_{\sigma}\right)$ in the $\frac{1}{N}$-limit, and we shall show that for factorizable initial conditions the fluctuations of intensive observables converge to a Gaussian stochastic process, rather similarly as it has been shown in [8] in a quantum mechanical context.

Acknowledgement. The authors are grateful to Prof. R. Jost for stimulating discussions.

\section{§2. The Vlasov Dynamics}

In this section we shall prove global existence, uniqueness and regularity of solutions of the Newtonian Equations (1.1), and we shall establish the connection between $z\left(t, a, \mu^{f}\right)$ and solutions $f(t, a)$ of the Vlasov equation.

Let $\mathscr{M}$ be the set of real Borel measures $\mu$ on $\mathbb{R}^{6}$ with $|\mu|<\infty$ and $\mathscr{M}_{+}^{1}$ the subset of probability measures. Let $C_{b}^{K}$ be the set of all potentials $\phi(q)=\phi(-\underline{q})$ with continuous and bounded derivatives up to order $k$ and $C_{b u}^{k}$ the subset with uniformly continuous derivatives.

Theorem 2.1. Let $\phi \in C_{b}^{2}$ and $\underline{F}=-\underline{\nabla} \phi$. Then (1.1) has a unique solution $z(t, a, \mu)$ for all $(t, a) \in \mathbb{R}^{7}$ and $\mu \in \mathscr{M} . z(t, a, \mu)$ is $C^{1}$ in $(t, a)$ and weakly continuous in $\mu$, uniformly for $a \in \mathbb{R}^{6}$ and bounded sets in $t$. The mapping $z(t, \mu): a \rightarrow z(t, a, \mu)$ is canonical and $T_{t}:(a, \mu) \rightarrow\left(z(t, a, \mu), \mu \circ z(t, \mu)^{-1}\right)$ is a 1-parameter group.

Proof. Let $T>0$ and let $B_{T}$ be the Banach space of all continuous bounded mappings $\underline{g}:[-1,1] \times \mathbb{R}^{6} \rightarrow \mathbb{R}^{3}$ with the norm

$$
\|\underline{g}\|_{T}=\sup _{|t| \leqq T, a \in \mathbb{R}^{6}}\|\underline{g}(t, a)\| .
$$

Let $U(\mu): B_{T} \rightarrow B_{T}$ be the mapping

$$
\begin{aligned}
& U(\mu, \underline{g})(t, a) \\
& \left.=\int_{0}^{t} d s \int_{0}^{s} d r \int \mu\left(d a^{\prime}\right) \underline{F}\left[\underline{g}(r, a)+\underline{q}+r \underline{p}-\underline{g}\left(r, a^{\prime}\right)-\underline{q}^{\prime}-r \underline{p}^{\prime}\right)\right] .
\end{aligned}
$$

Then $\underline{x}(t, a, \mu)=\underline{q}+\underline{p} t+\underline{g}(t, a, \mu)$ establishes a $1-1$ correspondence between solutions of (1.1) and fixed points

$$
\underline{g}(\cdot, \mu)=U(\mu, \underline{g}(\cdot, \mu))
$$

For $\phi \in C_{b}^{2}$ there exist constants $c_{1}, c_{2}$ such that

$$
\begin{aligned}
& \|U(\mu, \underline{g})\|_{T} \leqq c_{1} T^{2}|\mu| \\
& \left\|U\left(\mu, \underline{g}_{1}\right)-U\left(\mu, \underline{g}_{2}\right)\right\|_{T} \leqq c_{2} T^{2}\left\|\underline{g}_{1}-\underline{g}_{2}\right\|_{T}|\mu| .
\end{aligned}
$$

For small $T, U(\mu)$ is a contraction mapping and hence there exists a unique solution $z(t, a, \mu)$ which is $C^{1}$ in $(t, a)$ with uniformly bounded derivatives w.r.t. $a$. The 
mapping $z(t, \mu)$ is canonical, since $z(t, a, \mu)$ is a solution of the canonical equations

$$
\begin{aligned}
& \dot{x}=\frac{\partial H}{\partial \underline{y}}, \quad \dot{y}=-\frac{\partial H}{\partial \underline{x}} \\
& H(t, \underline{x}, \underline{y})=\frac{1}{2}\|\underline{y}\|^{2}+\int \mu\left(d a^{\prime}\right) \phi\left(\underline{x}-\underline{x}\left(t, a^{\prime}, \mu\right)\right) .
\end{aligned}
$$

Hence $\operatorname{Det} D z(t, a, \mu)=1$ and $\mu(t)=\mu \circ z(t, \mu)^{-1} \in \mathscr{M}$. The composition law $T_{t+s}$ $=T_{t} \circ T_{s}$ holds, if $z(t+s, \mu)=z(t, \mu(s)) \circ z(s, \mu)$ or if

$$
z(t+s, a, \mu)=z(t, z(s, a, \mu), \mu(s)) .
$$

Since $|\mu|=|\mu(s)|$, both sides are well-defined for $\max \{|s|,|t|,|s+t|\}<T$. For $t=0,(2.6)$ is true and both sides satisfy the same differential equation. This proves (2.6) and allows one to extend $z(t, \mu)$ for all $t \in \mathbb{R}$.

Consider now a sequence $\mu_{N} \in \mathscr{M}$ which converges to $\mu$ in the $w^{*}$ sense. Then uniform convergence $z\left(t a, \mu_{N}\right) \rightarrow z(t, a, \mu)$ holds, if we can show that the fixed points $\underline{g}\left(\mu_{N}\right)$ and $\underline{g}(\mu)$ satisfy $\left\|\underline{g}\left(\mu_{N}\right)-\underline{g}(\mu)\right\|_{T} \rightarrow 0$. Now, for sufficiently small $T$ and large $N$

$$
\begin{aligned}
& \left\|\underline{g}\left(\mu_{N}\right)-\underline{g}(\mu)\right\|_{T} \\
& \leqq\left\|U\left(\mu_{N}, \underline{g}\left(\mu_{N}\right)\right)-U\left(\mu_{N}, \underline{g}(\mu)\right)\right\|_{T}+\left\|U\left(\mu_{N}, \underline{g}(\mu)\right)-U(\mu, \underline{g}(\mu))\right\|_{T} \\
& \leqq \frac{1}{2}\left\|\underline{g}\left(\mu_{N}\right)-\underline{g}(\mu)\right\|_{T}+\left\|U\left(\mu_{N}, \underline{g}(\mu)\right)-U(\mu, \underline{g}(\mu))\right\|_{T},
\end{aligned}
$$

and hence we have to show that the last term converges to zero. If $\mu_{N} \rightarrow \mu$ in the $w^{*}$ sense, then $\left\|\mu_{N}-\mu\right\|_{B L}^{*} \rightarrow 0[10]$, where

$$
\begin{aligned}
& \|f\|_{B L}=\sup _{a \in \mathbb{R}^{6}}|f(a)|+\sup _{\substack{a_{1}, a_{2} \in \mathbb{R}^{6} \\
a_{1} \neq a_{2}}}\left|f\left(a_{1}\right)-f\left(a_{2}\right)\right|\left|a_{1}-a_{2}\right|^{-1} \\
& \|\mu\|_{B L}^{*}=\sup _{\|f\|_{B L}=1}\left|\int f(a) \mu(d a)\right| .
\end{aligned}
$$

Using the $B L$ norm in the variable $a^{\prime}$ one obtains

$$
\begin{aligned}
& \left\|U\left(\mu_{N}, \underline{g}(\mu)\right)-U(\mu, \underline{g}(\mu))\right\|_{T} \\
& \leqq\left\|\mu_{N}-\mu\right\|_{B L}^{*} \sup _{\substack{a \in \mathbb{R}^{6} \\
|t| \leqq T}} \int_{0}^{t} d s \int_{0}^{s} d r\left\|\underline{F}\left(\underline{g}(r, a, \mu)+\underline{q}+\underline{p} r-\underline{g}\left(r, a^{\prime}, \mu\right)-\underline{q}^{\prime}-\underline{p}^{\prime} r\right)\right\|_{B L} .
\end{aligned}
$$

The second factor on the right-hand side is bounded, because $g(r, a, \mu)$ has uniformly bounded derivatives w.r.t. $a$.

Theorem 2.2. For $\underline{F}=-\underline{\nabla} \phi, \phi \in C_{b}^{2}$ and $\mu \in \mathscr{M}_{+}^{1} \mu(t)$ is a weak solution of the Vlasov equation with $\mu(0)=\mu$. If $\mu=\mu^{f}$ with $0 \leqq f \in C^{1}\left(\mathbb{R}^{6}\right), \int f(a) d a=1$, then $f(t, a)$ $=f \circ z(t, \mu)^{-1}(a)$ is a strong solution of the Vlasov equation with $f(0, a)=f(a)$.

Remark. We have not proved the uniqueness of these solutions. 
Proof. Let $h \in C_{0}^{\infty}\left(\mathbb{R}^{6}\right)$. Then

$$
\begin{aligned}
& \frac{d}{d t} \int \mu(t, d a) h(a) \\
& =\frac{d}{d t} \int \mu(d a) h(z(t, a, \mu)) \\
& =\int \mu(d a)\left\{\frac{\partial h}{\partial \underline{x}}(z(t, a, \mu)) \cdot \underline{y}(t, a, \mu)\right. \\
& \left.\quad+\frac{\partial h}{\partial \underline{y}}(z(t, a, \mu)) \int \mu(d b) \underline{F}[\underline{x}(t, a, \mu)-\underline{x}(t, b, \mu)]\right\} \\
& =\int \mu(t, d z)\left\{\frac{\partial h}{\partial \underline{x}}(z) \cdot \underline{y}+\frac{\partial h}{\partial \underline{y}}(z) \int \mu\left(t, d z^{\prime}\right) \underline{F}\left[\underline{x}-\underline{x}^{\prime}\right]\right\} .
\end{aligned}
$$

If $\mu=\mu^{f}$, then Det $D z(t, a, \mu)=1$ implies $\mu^{f}(t)=\mu^{f(t)}$, where $f\left(t, z\left(t, a, \mu^{f}\right)\right)=f(a)$. Hence

$$
\begin{aligned}
0= & \frac{d}{d t} f\left(t, z\left(t, a, \mu^{f}\right)\right) \\
= & \frac{\partial f}{\partial t}\left(t, z\left(t, a, \mu^{f}\right)\right)+\frac{\partial f}{\partial \underline{x}}\left(t, z\left(t, a, \mu^{f}\right)\right) \underline{y}\left(t, a, \mu^{f}\right) \\
& +\frac{\partial f}{\partial \underline{y}}\left(t, z\left(t, a, \mu^{f}\right)\right) \int f\left(a^{\prime}\right) d a^{\prime} \underline{F}\left[\underline{x}\left(t, a, \mu^{f}\right)-\underline{x}\left(t, a^{\prime}, \mu^{f}\right)\right] .
\end{aligned}
$$

Now the last integral equals

$$
\int d z^{\prime} f\left(t, z^{\prime}\right) \underline{F}\left[\underline{x}\left(t, a, \mu^{f}\right)-\underline{x}^{\prime}\right]
$$

and the substitution $a=z(t, \mu)^{-1}(z)$ leads to (1.5).

In the next section we shall need stronger regularity properties of $z(t, a, \mu)$ in $\mu$, which we can prove under additional smoothness assumptions on $\phi$ :

Theorem 2.3. Let $k \in \mathbb{N}, \underline{F}=-\underline{\nabla} \phi$ and $\phi \in C_{b u}^{1+k}$, then $z(t, a, \mu)$ is $k$ times continuously differentiable w.r.t. $\mu$, where for $\mu, v \in \mathscr{M}$

$$
\begin{aligned}
& \lim _{t \rightarrow 0} t^{-1}\{z(t, a, \mu+t v)-z(t, a, \mu)\} \\
& \equiv \int v(d b) D^{1} z(t, a, b, \mu)
\end{aligned}
$$

and $D^{j} z\left(t, a, b_{1}, \ldots, b_{j}, \mu\right)$ similarly. For every finite $t D^{j} z$ is uniformly bounded

$$
\sup _{\substack{a, b_{1} \\|\mu| \leqq i}}\left|D^{j} z\left(t, a, b_{1}, \ldots, b_{j}, \mu\right)\right| \leqq C^{j}(t) \quad j=1 \ldots k
$$

and

$$
D^{1} z(t, a, b, \mu)=\left(D^{1} \underline{x}(t, a, b, \mu), D^{1} \underline{y}(t, a, b, \mu) \equiv D^{1} \underline{\dot{x}}(t, a, b, \mu)\right)
$$


is $C^{k}$ in $t$ and $C^{k-1}$ in $a, b$ and is the solution of the linearization of (1.1) around $z(t, a, \mu)$ :

$$
\begin{aligned}
D^{1} \underline{x}(t, a, b, \mu)= & \int_{0}^{t} d s \int_{0}^{s} d r \underline{F}[\underline{x}(r, a, \mu)-\underline{x}(r, b, \mu)] \\
& +\int_{0}^{t} d_{S} \int_{0}^{s} d r \int \mu(d c) \frac{\partial \underline{\underline{F}}}{\partial \underline{x}}[\underline{x}(r, a, \mu)-\underline{x}(r, c, \mu)] \\
& \cdot\left\{D^{1} \underline{x}(r, a, b, \mu)-D^{1} \underline{x}(r, c, b, \mu)\right\} .
\end{aligned}
$$

Proof. The well-known proof for ordinary differential equations using Gronwall's inequality (see e.g. [9], p. 302) can be applied without complications. The boundedness of $D^{j} z$ follows immediately from the linear Volterra equation for $D^{j} z$, by applying $D^{j-1}$ to $(2.15)$.

\section{§ 3. The Weak Coupling Limit for Infinitely Many Classical Particles}

In this section we shall investigate the weak coupling limit for a classical $N$-particle system with pair potential $N^{-1} \phi$. First we shall consider pure initial states:

Theorem 3.1. Let $\underline{F}=-\underline{\nabla} \phi$ and $\phi \in C_{b}^{2}$. Let $z_{n}\left(t, \alpha_{N}\right)$ be the solution of

$$
\begin{aligned}
& \ddot{\ddot{x}}_{n}\left(t, \alpha_{N}\right)=N^{-1} \sum_{m=1}^{N} \underline{F}\left[\underline{x}_{n}\left(t, \alpha_{N}\right)-\underline{x}_{m}\left(t, \alpha_{N}\right)\right] \\
& \left(\underline{x}_{n}\left(0, \alpha_{N}\right), \underline{\dot{x}}_{n}\left(0, \alpha_{N}\right)\right)=a_{n} \quad 1 \leqq n \leqq N .
\end{aligned}
$$

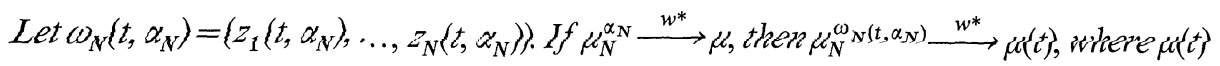
$=\mu \circ z(t, \mu)^{-1}$ is a weak solution of the Vlasov equation.

Proof. We use the fact that $z_{n}\left(t, \alpha_{N}\right)=z\left(t, a_{n}, \mu_{N}^{\alpha_{N}}\right)$ is the solution of (1.1) and estimate for $h \in C_{b}^{0}\left(\mathbb{R}^{6}\right)$

$$
\begin{aligned}
& \left|\int \mu_{N}^{\omega_{N}\left(t, \alpha_{N}\right)}(d a) h(a)-\int \mu(t, d a) h(a)\right| \\
& =\left|N^{-1} \sum_{n=1}^{N} h\left[z\left(t, a_{n}, \mu_{N}^{\alpha_{N}}\right)\right]-\int \mu(d a) h[z(t, a, \mu)]\right| \\
& =\left|\int \mu_{N}^{\alpha_{N}}(d a) h\left[z\left(t, a, \mu_{N}^{\alpha_{N}}\right)\right]-\int \mu(d a) h[z(t, a, \mu)]\right| \\
& \leqq\left|\int\left(\mu_{N}^{\alpha}-\mu\right)(d a) h[z(t, a, \mu)]\right|+\left|\int \mu_{N}^{\alpha_{N}}(d a)\left\{h\left[z\left(t, a, \mu_{N}^{\alpha_{N}}\right)\right]-h[z(t, a, \mu)]\right\}\right| .
\end{aligned}
$$

By assumption, the first term tends to zero. Since $\mu_{N}^{\alpha_{N}}$ converges weakly, there exists for every $\varepsilon>0$ a compact $K(\varepsilon) \subset \mathbb{R}^{6}$ such that $\mu_{N}^{\alpha_{N}}(K(\varepsilon)) \geqq 1-\varepsilon$. On $K(\varepsilon), h$ is uniformly continuous and the supremum of $h\left[z\left(t, a, \mu_{N}^{\alpha_{N}}\right)\right]-h[z(t, a, \mu)]$ tends to zero by Theorem 2.1. 
Corollary 3.2. If $\mu_{N}^{\alpha_{N}} \stackrel{w^{*}}{\longrightarrow} \mu$ and $h \in C_{b}^{0}\left(\mathbb{R}^{6 s}\right)$ then the "intensive observables"

$$
O_{n}^{t}\left(\alpha_{N}\right) \equiv N^{-s} \sum_{n_{1} \ldots n_{s}=1}^{N} h\left[z_{n_{1}}\left(t, \alpha_{N}\right), \ldots, z_{n_{s}}\left(t, \alpha_{N}\right)\right]
$$

converge:

$$
\lim _{N \rightarrow \infty} O_{n}^{t}\left(\alpha_{N}\right)=\int \mu\left(d a_{1}\right) \ldots \mu\left(d a_{s}\right) h\left[z\left(t, a_{1}, \mu\right), \ldots, z\left(t, a_{s}, \mu\right)\right] .
$$

Next we shall investigate the time evolution of symmetric product measures $\tilde{\mu}\left(d \alpha_{N}\right)$ $=\prod_{n} \mu\left(d a_{n}\right)$ on the $N$-particle phase space with $\mu \in \mathscr{M}_{+}^{1}$. Let $\tilde{\mu}_{t}\left(d \alpha_{N}\right)$ be defined by $\int \tilde{\mu}_{t}\left(d \alpha_{N}\right) g\left(\alpha_{N}\right)=\int \tilde{\mu}\left(d \alpha_{N}\right) g\left(\omega_{N}\left(t, \alpha_{N}\right)\right)$.

We shall see that "molecular chaos" propagates for $N \rightarrow \infty$, if we only look at intensive observables of the type $g\left(\alpha_{N}\right)=O_{h}\left(\alpha_{N}\right)$ :

Theorem 3.3. Let $h \in C_{b}^{0}\left(\mathbb{R}^{6}\right), \mu \in \mathscr{M}_{+}^{1}$ and $\tilde{\mu}_{t}\left(d \alpha_{N}\right)$ be the solution of (3.1) with $\tilde{\mu}_{0}\left(d \alpha_{N}\right)$ $=\prod_{n} \mu\left(d a_{n}\right)$. Then

$$
\lim _{N \rightarrow \infty} \int O_{h}\left(\alpha_{N}\right) \tilde{\mu}_{t}\left(d \alpha_{N}\right)=\int h\left(a_{1}, \ldots, a_{s}\right) \prod_{\sigma=1}^{s} \mu\left(t, d a_{\sigma}\right)
$$

where $\mu(t)=\mu \circ z(t, \mu)^{-1}$ is a weak solution of the Vlasov equation.

Proof. At time $t=0,(3.5)$ is obvious:

$$
\begin{aligned}
& N^{-s} \sum_{n_{1} \ldots n_{s}=1}^{N} \int h\left(a_{n_{1}}, \ldots, a_{n_{s}}\right) \tilde{\mu}\left(d \alpha_{N}\right) \\
& =\int h\left(a_{1}, \ldots, a_{s}\right) \prod_{\sigma=1}^{s} \mu\left(d a_{\sigma}\right)+O\left(N^{-1}\right) .
\end{aligned}
$$

For $t \neq 0$ we shall use (3.4) and the strong law of large numbers : Let $\mathscr{B}^{6}$ be the Borel $\sigma$-algebra on $\mathbb{R}^{6}$ and $(\Omega, \mathscr{B})$ be the probability space $\prod_{n=1}^{\infty}\left(\mathbb{R}^{6}, \mathscr{B}^{6}\right)$ with the product measure $\tilde{\mu}(d \alpha)=\prod_{n=1}^{\infty} \mu\left(d a_{n}\right)$. Here $\alpha=\left(a_{1}, \ldots, a_{n}, \ldots\right) \in \Omega$ and $\alpha_{N}$ denotes its projection on $\mathbb{R}^{6 N}$. For every $\mu$-continuous set $\Delta \subset \mathscr{B}^{6}$ [i.e. $\mu(\partial \Delta)=0$ ] with characteristic function $\chi_{\Delta}$, the independent random variables in $(\Omega, \mathscr{B}, \tilde{\mu}) \chi_{\Delta}^{n}(\alpha)=\chi_{\Delta}\left(a_{n}\right)$ have mean $\mu(\Delta)$ and variance $\mu(\Delta)-\mu(\Delta)^{2}$. Hence

$$
\mu_{N}^{\alpha}(\Delta)=N^{-1} \sum_{n=1}^{N} \chi_{\Delta}^{n}(\alpha) \underset{N \rightarrow \infty}{\longrightarrow} \mu(\Delta), \tilde{\mu} \quad \text { a.e. }
$$

Since $\mu \in \mathscr{M}_{+}^{1}$, there exists a countable set $\mathfrak{A}$ of intervals

$$
\left(a_{n}, b_{n}\right]=\left\{z \in \mathbb{R}^{6} \mid a_{n}^{i}<z_{n}^{i} \leqq b_{n}^{i}, 1 \leqq i \leqq 6\right\},
$$

which is closed under finite intersections, such that for every $z \in \mathbb{R}^{6}$ and every $\varepsilon>0$ there exists an $\left(a_{n}, b_{n}\right] \in \mathfrak{U}$ with $z \in\left(a_{n}, b_{n}\right) \subset\left\{z^{\prime}|| z-z^{\prime} \mid<\varepsilon\right\}$ and such that (3.7) holds. 
Since $\mathfrak{A}$ is countable there exists a set $Q \subset \Omega$ with $\tilde{\mu}(Q)=1$, such that $\mu_{N}^{\alpha}\left(\left(a_{n}, b_{n}\right]\right)$ $\rightarrow \mu\left(\left(a_{n}, b_{n}\right)\right)$ for all $\alpha \in Q$ and all $\left(a_{n}, b_{n}\right] \in \mathfrak{A}$. Hence $\mu_{N}^{\alpha}$ converges in the $w^{*}$-sense to $\mu$ ([11], p. 14) for all $\alpha \in Q$. Now

$$
\int \tilde{\mu}_{t}\left(d \alpha_{N}\right) O_{n}\left(\alpha_{N}\right)=\int \tilde{\mu}(d \alpha) O_{n}^{t}\left(\alpha_{N}\right) .
$$

For all $N$,

$$
\left|O_{n}^{t}\left(\alpha_{N}\right)\right| \leqq \sup \left|h\left(a_{1}, \ldots, a_{s}\right)\right|<\infty,
$$

and for $\alpha \in Q$

$$
O_{n}^{t}\left(\alpha_{N}\right) \rightarrow \int h\left(a_{1}, \ldots, a_{s}\right) \prod_{\sigma}^{s} \mu\left(t, d a_{\sigma}\right)
$$

by (3.4). Since $\tilde{\mu}(Q)=1$, one obtains (3.5) by the dominated convergence theorem.

For more general initial states, which do not factorize, one has the

Corollary 3.4. Let $\tilde{\pi}$ be a symmetric probability measure on $(\Omega, \mathscr{B}), \tilde{\pi}_{N}$ its projection on the $N$-particle space and $\tilde{\pi}_{N}^{t}$ its time translation by (3.1). Then

$$
\lim _{N \rightarrow \infty} \int \tilde{\pi}_{N}^{t}\left(d \alpha_{N}\right) O_{n}\left(\alpha_{N}\right)=\int \beta_{t}^{s}\left(d a_{1}, \ldots, d a_{s}\right) h\left(a_{1}, \ldots, a_{s}\right)
$$

where $\left\{\beta_{t}^{s}\right\}$ is a weak solution of the Vlasov hierarchy (1.9).

Proof. $\tilde{\pi}$ can be uniquely decomposed into product measures $\tilde{\mu}[12], \tilde{\pi}=\int \tilde{\sigma}(\tilde{\pi}, d \tilde{\mu}) \tilde{\mu}$. Hence

$$
\begin{aligned}
& \int \tilde{\pi}_{N}^{t}\left(d \alpha_{N}\right) O_{n}\left(\alpha_{N}\right)=\int \tilde{\pi}(d \alpha) O_{n}^{t}\left(\alpha_{N}\right) \\
& =\int \tilde{\sigma}(\tilde{\pi}, d \tilde{\mu}) \int \tilde{\mu}(d \alpha) O_{n}^{t}\left(\alpha_{N}\right) \\
& \quad \underset{N \rightarrow \infty}{\longrightarrow} \int \tilde{\sigma}(\tilde{\pi}, d \tilde{\mu}) \int \prod_{\sigma=1}^{s} \mu\left(t, d a_{\sigma}\right) h\left(a_{1}, \ldots, a_{s}\right) \\
& =\int \beta_{t}^{s}\left(d a_{1}, \ldots, d a_{s}\right) h\left(a_{1}, \ldots, a_{s}\right)
\end{aligned}
$$

by Theorem 3.3 and the dominated convergence theorem. Since $\left\{\prod_{\sigma=1}^{s} \mu\left(t, d a_{s}\right)\right\}$ is a weak solution of the linear Vlasov hierarchy, the convex combination $\left\{\beta_{t}^{s}\right\}$ is also a solution with initial condition $\beta_{0}^{s}=\tilde{\pi}_{s}$.

By the law of large numbers the solutions $z\left(t, a, \mu_{N}^{\alpha}\right)$ of (3.1) converge to $z(t, a, \mu)$ in the probability space $(\Omega, \mathscr{B}, \tilde{\mu})$. We shall now establish a central limit theorem for the fluctuations

$$
\zeta_{N}\left(t, a, \mu_{N}^{\alpha}\right)=\sqrt{N}\left(z\left(t, a, \mu_{N}^{\alpha}\right)-z(t, a, \mu)\right)
$$

$\zeta_{N}\left(t, a, \mu_{N}^{\alpha}\right)$ describes the deviations of a deterministic $N$-particle orbit from its "mean field" approximation. 
Theorem 3.5. Let $\underline{F}=-\underline{\nabla} \phi$ with $\phi \in C_{b u}^{4}$. Then

$$
\begin{aligned}
& \lim _{N \rightarrow \infty} \int \tilde{\mu}(d \alpha) \exp \left(i \sum_{k=1}^{K} u_{k} \zeta_{N}\left(t_{k}, e_{k}, \mu_{N}^{\alpha}\right)\right) \\
& =\exp \left(-\frac{1}{2} \sum_{k, k^{\prime}=1}^{K} Q_{k k^{\prime}} u_{k} u_{k^{\prime}}\right) \\
& =E \exp \left(i \sum_{k=1}^{K} u_{k} \zeta\left(t_{k}, e_{k}, \mu\right)\right), \\
& Q_{k k^{\prime}}=\int \mu(d b) D^{1} z\left(t_{k}, e_{k}, b, \mu\right) D^{1} z\left(t_{k^{\prime}}, e_{k^{\prime}}, b, \mu\right) \\
& \quad-\prod_{\chi \in\left\{k, k^{\prime}\right\}} \int \mu\left(d b_{\varkappa}\right) D^{1} z\left(t_{\varkappa}, e_{\varkappa}, b_{\varkappa}, \mu\right)
\end{aligned}
$$

The convergence in (3.11) is uniform, if the $e_{k}$ vary over $\mathbb{R}^{6}$ and the $t_{k}$ and $u_{k}$ over bounded regions. The Gaussian stochastic process $\zeta=(\xi, \xi)$ with mean zero and variance (3.12) can be represented in terms of the Gaussian process $\varphi(\mu, d b)$ on $\mathbb{R}^{6}$ with $E \varphi(\mu, \Delta)=0, E \varphi\left(\mu, \Delta_{1}\right) \varphi\left(\mu, \Delta_{2}\right)=\mu\left(\Delta_{1} \cap \Delta_{2}\right)-\mu\left(\Delta_{1}\right) \mu\left(\Delta_{2}\right)$ :

$$
\zeta(t, a, \mu)=\int D^{1} z(t, a, b, \mu) \varphi(\mu, d b)
$$

and satisfies the stochastic differential equation

$$
\begin{aligned}
\underline{\xi}(t, a, \mu)= & \int \underline{F}[\underline{x}(t, a, \mu)-\underline{x}(t, b, \mu)] \varphi(\mu, d b) \\
& +\int \mu(d c) \frac{\partial \underline{F}}{\partial \underline{x}}[\underline{x}(t, a, \mu)-\underline{x}(t, c, \mu)] \\
& \cdot\{\xi(t, a, \mu)-\xi(t, c, \mu)\},
\end{aligned}
$$

$\xi(0, a, \mu)=\dot{\xi}(0, a, \mu)=0$.

Proof. As a consequence of Theorem 2.3 the following manipulations are legitimate:

$$
\begin{aligned}
& \exp \left(i \sum_{k=1}^{K} u_{k} \zeta_{N}\left(t_{k}, e_{k}, \mu_{N}^{\alpha}\right)\right) \\
& =\exp \left(i \sum_{k=1}^{K} u_{k} \sqrt{N} \int_{0}^{1} d r \int D^{1} z\left(t_{k}, e_{k}, b, r \mu_{N}^{\alpha}+(1-r) \mu\right)\left(\mu_{N}^{\alpha}-\mu\right)(d b)\right) \\
& =\exp \left(i \sum_{k=1}^{K} u_{k} \sqrt{N} \int D^{1} z\left(t_{k}, e_{k}, b, \mu\right)\left(\mu_{N}^{\alpha}-\mu\right)(d b)\right) \\
& \quad+\int_{0}^{1} d s \frac{d}{d s} \exp \left[i \sum_{k=1}^{K} u_{k} \sqrt{N} \int D^{1} z\left(t_{k}, e_{k}, b, r s \mu_{N}^{\alpha}+(1-r s) \mu\right)\left(\mu_{N}^{\alpha}-\mu\right)(d b)\right]
\end{aligned}
$$

By the central limit theorem, the $\tilde{\mu}$-integral of the first term on the right side converges to $\exp \left(-\frac{1}{2} \sum Q_{k k^{\prime}} u_{k} u_{k^{\prime}}\right)$ as $N \rightarrow \infty$. After having carried out the $s$ - 
differentiation and having suppressed the inessential dependence on $u_{k}, t_{k}, e_{k}$, the second term takes the typical form

$$
\begin{aligned}
& \exp \left\{i \int f\left(a, \mu_{N}^{\alpha}\right) \sqrt{N}\left(\mu_{N}^{\alpha}-\mu\right)(d a)\right\} \\
& \cdot \sqrt{N} \int g\left(b, c, \mu_{N}^{\alpha}\right)\left(\mu_{N}^{\alpha}-\mu\right)(d b)\left(\mu_{N}^{\alpha}-\mu\right)(d c) .
\end{aligned}
$$

Since $\phi \in C_{b u}^{4}, f$ and $g$ are differentiable w.r.t. $\mu$ and $D^{1} f(b, a, \mu), D^{2} f(a, b, c, \mu)$ and $D^{1} g(a, b, c, \mu)$ are continuous and uniformly bounded in $a, b, c$. Now

$$
\begin{aligned}
& \sqrt{N} \int g\left(b, c, \mu_{N}^{\alpha}\right)\left(\mu_{N}^{\alpha}-\mu\right)(d b)\left(\mu_{N}^{\alpha}-\mu\right)(d c) \\
& =N^{-3 / 2} \sum_{m, n=1}^{N}\left\{g\left(a_{m}, a_{n}, \mu_{N}^{\alpha}\right)-\int g\left(b, a_{n}, \mu_{N}^{\alpha}\right) \mu(d b)\right. \\
& \left.\quad-\int g\left(a_{m}, c, \mu_{N}^{\alpha}\right) \mu(d c)+\int g\left(b, c, \mu_{N}^{\alpha}\right) \mu(d b) \mu(d c)\right\} .
\end{aligned}
$$

The sum over the $N$ terms with $m=n$ is $O\left(N^{-1 / 2}\right)$ and can be dropped in (3.16) for $N \rightarrow \infty$. For $m \neq n$ we consider $m=1, n=2$. Let ' $\mu_{N}^{\alpha}=N^{-1} \sum_{n=3}^{N} \delta_{a_{n}}$. Then

$$
g\left(a, b, \mu_{N}^{\alpha}\right)=g\left(a, b, \mu_{N}^{\alpha}\right)+N^{-1} \sum_{n=1}^{2} D^{1} g\left(a, b, a_{n}\right)+O\left(N^{-2}\right),
$$

and up to an error of $O\left(N^{-5 / 2}\right)$ in the sum (3.17) we can replace $g\left(a, b, \mu_{N}^{\alpha}\right)$ by $g\left(a, b,{ }^{\prime} \mu_{N}^{\alpha}\right)$. The factor multiplying the $(1,2)$-term can also be simplified:

$$
\begin{aligned}
& \exp i N^{-1 / 2} \sum_{n=1}^{N}\left\{f\left(a_{n}, \mu_{N}^{\alpha}\right)-\int f\left(a, \mu_{N}^{\alpha}\right) \mu(d a)\right\} \\
& =\left(1+i N^{-1 / 2} \sum_{n=1}^{2}\left\{f\left(a_{n},{ }^{\prime} \mu_{N}^{\alpha}\right)-\int f\left(a,{ }^{\prime} \mu_{N}^{\alpha}\right) \mu(d a)\right\}+O\left(N^{-1}\right)\right) \\
& \quad \cdot\left[\operatorname { e x p } i N ^ { - 1 / 2 } \sum _ { n = 3 } ^ { N } \{ f ( a _ { n } , { } ^ { \prime } \mu _ { N } ^ { \alpha } ) - \int f ( a , { } ^ { \prime } \mu _ { N } ^ { \alpha } ) \mu ( d a ) \} \cdot \left(1+O\left(N^{-1}\right)\right.\right. \\
& \left.\left.\quad+i N^{-3 / 2} \sum_{n=3}^{N} \sum_{m=1}^{2} \cdot\left\{D^{1} f\left(a_{n}, a_{m},{ }^{\prime} \mu_{N}^{\alpha}\right)-\int D^{1} f\left(a, a_{m},{ }^{\prime} \mu_{N}^{\alpha}\right) \mu(d a)\right\}\right)\right] .
\end{aligned}
$$

(3.19) is a sum of terms $O(1)+O\left(N^{-1 / 2}\right)+O\left(N^{-1}\right)$. It is easy to see that

$$
\begin{aligned}
0= & \int \tilde{\mu}(d \alpha)\left\{O(1)+O\left(N^{-1 / 2}\right)\right\} N^{-3 / 2}\left\{g\left(a_{1}, a_{2},{ }^{\prime} \mu_{N}^{\alpha}\right)\right. \\
& -\int g\left(b, a_{2},{ }^{\prime} \mu_{N}^{\alpha}\right) \mu(d b)-\int g\left(a_{1}, c,{ }^{\prime} \mu_{N}^{\alpha}\right) \mu(d c) \\
& \left.+\int g\left(b, c,{ }^{\prime} \mu_{N}^{\alpha}\right) \mu(d b) \mu(d c)\right\} .
\end{aligned}
$$

For, the $O(1)$ term is independent of $a_{1}$ and $a_{2}$, and the $O\left(N^{-1 / 2}\right)$ term is a sum of a term independent of $a_{1}$ and one of $a_{2}$, and therefore either $\int \mu\left(d a_{1}\right)$ or $\int \mu\left(d a_{2}\right)$ gives zero. The remainder is again $O\left(N^{-5 / 2}\right)$ and there are $N(N-1)$ terms of this type in (3.16), which are together $O\left(N^{-1 / 2}\right)$. This proves the convergence of (3.11). The identification of the Gaussian stochastic process uses (2.15). 
By similar methods one can prove that for $h_{k} \in C_{b u}^{3}\left(\mathbb{R}^{6 s}\right)$, the fluctuations of the intensive observables converge:

$$
\begin{aligned}
& \lim _{N \rightarrow \infty} \int \tilde{\mu}(d \alpha) \exp i \sum_{k=1}^{K}\left\{O_{h_{k}}^{t_{k}}\left(\mu_{N}^{\alpha}\right)-O_{h_{k}}^{t_{k}}(\mu)\right\} \sqrt{N} \\
& =E \exp i \sum_{k=1}^{K} D O_{h_{k}}^{t_{k}}(b, \mu) \varphi(\mu, d b),
\end{aligned}
$$

where

$$
\begin{aligned}
D O_{h}^{t}(b, \mu)= & \sum_{\sigma=1}^{s}\left\{\int \prod_{\substack{\tau=1 \\
\tau \neq \sigma}}^{s} \mu\left(d a_{\tau}\right) h\left(z\left(t, a_{1}, \mu\right), \ldots z(t, b, \mu), \ldots z\left(t, a_{s}, \mu\right)\right)\right. \\
& \left.+\int \prod_{\tau=1}^{s} \mu\left(d a_{\tau}\right) \frac{\partial h}{\partial z_{\sigma}}\left(z\left(t, a_{1}, \mu\right), \ldots z\left(t, a_{s}, \mu\right)\right) D^{1} z\left(t, a_{\sigma}, b, \mu\right)\right\} .
\end{aligned}
$$

One can also prove the convergence of moments, as for instance in

Corollary 3.6. If $\underline{F}=-\underline{\nabla} \phi$ and $\phi \in C_{0}^{\infty}\left(\mathbb{R}^{3}\right)$. Then for all $K, t_{1}, \ldots t_{K}, e_{1}, \ldots e_{K}$,

$$
\lim _{N \rightarrow \infty} \int \tilde{\mu}(d \alpha) \prod_{k=1}^{K} \zeta_{N}\left(t_{k}, e_{k}, \mu_{N}^{\alpha}\right)=E \prod_{k=1}^{K} \zeta\left(t_{k}, e_{k}, \mu\right) .
$$

Proof. Similarly as in (3.15) we have

$$
\begin{aligned}
& \int \tilde{\mu}(d \alpha) \prod_{k=1}^{K} \zeta_{N}\left(t_{k}, e_{k}, \mu_{N}^{\alpha}\right)=N^{-K / 2} \sum_{n_{1}, \ldots n_{K}=1}^{N} F_{N}\left(n_{1}, \ldots n_{K}\right) \\
& =N^{-K / 2} \sum_{j=0}^{K} N(N-1) \ldots(N-j+1) \sum_{\underline{\alpha} \in \mathscr{P}(K, j)} \tilde{F}_{N}(\underline{\alpha})
\end{aligned}
$$

with

$$
\begin{aligned}
& F_{N}\left(n_{1}, \ldots n_{K}\right) \equiv \tilde{F}_{N}(\underline{\alpha}) \\
= & \int \tilde{\mu}(d \alpha) \prod_{k=1}^{K} \int_{0}^{1} d s_{k}\left\{D^{1} z\left(t_{k}, e_{k}, a_{n_{k}}, s_{k} \mu_{N}^{\alpha}+\left(1-s_{k}\right) \mu\right)-\int D^{1} z\left(t_{k}, e_{k}, a, s_{k} \mu_{N}^{\alpha}+\left(1-s_{k}\right) \mu\right) \mu(d a)\right\}
\end{aligned}
$$

where every $\left(n_{1}, \ldots n_{K}\right)$ defines a partition

$$
\underline{\alpha}=\left(\alpha_{1}^{1}, \ldots \alpha_{\beta_{1}}^{1}\right)\left(\alpha_{1}^{2}, \ldots \alpha_{\beta_{2}}^{2}\right) \ldots\left(\alpha_{1}^{j} \ldots \alpha_{\beta_{j}}^{j}\right)
$$

of $(1, \ldots K)$ in subsets with equal indices

$$
\begin{aligned}
& n_{\alpha_{s}^{r}}=n_{\alpha_{t}^{r}}, \quad 1 \leqq r \leqq j, \quad 1 \leqq s, \quad\left[t \leqq \beta_{r}\right. \\
& n_{\alpha_{s}^{r}} \neq n_{\alpha_{t}^{u}} \quad \text { for } \quad r \neq u .
\end{aligned}
$$

By Theorem $2.3 \tilde{F}_{N}(\underline{\alpha})$ is uniformly bounded in $N$ so we can restrict the summation over $j$ in (3.24) in the limit $N \rightarrow \infty$ to $j \geqq K / 2$. 


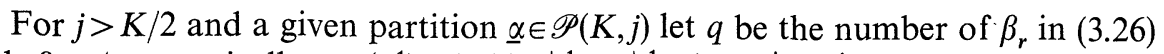
with $\beta_{r}=1$, so typically $\underline{\alpha}=\left(\alpha_{1}^{1}\right) \ldots\left(\alpha_{1}^{q}\right)\left(\alpha_{1}^{q+1}, \alpha_{2}^{q+1} \cdots\right) \cdots\left(\alpha_{1}^{j} \cdots \alpha_{\beta}^{j}\right)$, then

$$
q \geqq 2 j-K \text {. }
$$

By expanding the integrand in (3.25) in a Taylor series of order $q$ around

$$
' \mu_{N}^{\alpha}=N^{-1} \sum_{\substack{n=1 \\ n \neq n l, 1 \leqq l \leqq q}}^{N} \delta_{a_{n}}
$$

the $\tilde{\mu}(d \alpha)$-integration over every term of order $p<q$ in this expansion gives zero, the remainder is again by Theorem 2.3 of order $O\left(N^{-q}\right)$, which implies

$$
\left|\tilde{F}_{N}(\underline{\alpha})\right| \leqq c(K, j) N^{-(2 j-K)}
$$

for $\underline{\alpha} \in \mathscr{P}(K, j)$ and $j>K / 2$.

Hence the only surviving partitions are those with $j=K / 2$, so $K$ must be even to give a nonvanishing contribution. By the same argument as before, all partition with $q \geqq 1$ also vanish in the limit $N \rightarrow \infty$, so we end up with partitions of $K / 2$ pairs of identical indices, which are pairwise different from one another, typically

$$
\begin{aligned}
& n_{1}=n_{2}, \ldots n_{K-1}=n_{K}, \\
& n_{2 r} \neq n_{2 s} \text { for } r \neq s .
\end{aligned}
$$

In every of these terms in the sum over $\underline{\alpha}$ in (3.24) we can replace up to $O\left(N^{-1}\right) \mu_{N}^{\alpha}$ by $' \mu_{N}^{\alpha}$.

The $\tilde{\mu}(d \alpha)$-integration yields

$$
\begin{aligned}
& \int \tilde{\mu}(d \alpha) \prod_{\sigma=1}^{K / 2}\left\{\int \mu\left(d a_{2 \sigma}\right) \prod_{\tau=2 \sigma-1}^{2 \tau} D^{1} z\left(t_{\tau}, e_{\tau}, a_{2 \sigma}, s_{\tau}{ }^{\prime} \mu_{N}^{\alpha}+\left(1-s_{\tau}\right) \mu\right)\right. \\
& \left.-\prod_{\tau=2 \sigma-1}^{2 \sigma} \int \mu\left(d a_{\tau}\right) D^{1} z\left(t_{\tau}, e_{\tau}, a_{\tau},{ }^{\prime}{ }_{\tau}^{\prime} \mu_{N}^{\alpha}+\left(1-s_{\tau}\right) \mu\right)\right\} .
\end{aligned}
$$

As we keep $K$ fixed, we have as in Theorem 3.3

$$
w^{*}-\lim _{N \rightarrow \infty}{ }^{\prime} \mu_{N}^{\alpha}=\mu \quad \tilde{\mu} \quad \text { a.e. }
$$

The continuity property of $D^{1} z(t, a, b, \mu)$ with respect to $\mu$ and the dominated convergence theorem prove then the convergence of (3.23).

\section{§4. Conclusion}

The weak coupling limit has the following physically rather attractive interpretation: Take

$$
\begin{aligned}
H_{N} & =\sum_{n=1}^{N} \frac{p_{n}^{2}}{2 m_{N}}+\sum_{1 \leqq m<n \leqq N} \phi\left(q_{m}-q_{n}\right), \\
m_{N} & =N^{-1}, \quad \tau_{N}=N t .
\end{aligned}
$$


Then one obtains the time evolution (3.1) in the rescaled time $\tau_{N}$. In the limit $N \rightarrow \infty$, where the total mass $\mathrm{Nm}_{\mathrm{N}}$ stays finite, the Vlasov equation describes the continuum theory of infinitely small particles". The limit dynamics is time reversal invariant, and nevertheless molecular chaos propagates, as in the irreversible Boltzmann gas. These results can be easily generalized to canonical and non-canonical systems with weak many-body forces.

For the corresponding quantum mechanical problem, one obtains the Hartree dynamics in this weak coupling limit. This has been proved in [13] using a family of coherent states, but similar results hold for density matrices which are $N$-fold tensor products $P^{\otimes N}$ of a 1-particle state $P[14]$.

\section{References}

1. Vlasov, A. A.: J.E.T.P. 8, 291 (1938)

2. Kac, M.: Proc. $3^{\text {rd }}$ Berkeley Symp. Math. Stat. Prob. 3, 171 (1955)

3. McKean,H.P.: Comm. Pure Appl. Math. 28, 435 (1975)

4. Grünbaum,F.A.: Arch. Rat. Mech. Anal. 42, 323 (1971)

5. Grad,H.: In : Handbuch der Physik, Vol. XII. Berlin-Göttingen-Heidelberg: Springer 1958

6. Lanford,O.E.,III: In: Dynamical systems, theory and applications (ed. J.Moser). BerlinHeidelberg-New York: Springer 1975

7. King, F.: Thesis, Univ. of California, Berkeley (1976)

8. Hepp,K., Lieb,E.H. : Helv. Phys. Acta 46, 573 (1973)

9. Hirsch, M.W., Smale,S. : Differential equations, dynamical systems, and linear algebra. New York: Academic Press 1974

10. Dudley, R.M.: Studia Math. 27, 251 (1966)

11. Billingsley,P.: Convergence of probability measures. New York: Wiley 1968

12. Hewitt, E., Savage,L.: Trans. Amer. Math. Soc. 80, 470 (1955)

13. Hepp, K.: Commun. math. Phys. 35, 265 (1974)

14. Battle, G.: Dynamics and phase transitions for a continuous system of quantum particles in a box. Preprint

Communicated by J. L. Lebowitz 
